\title{
INEQUALITIES FOR $\max \left|S_{\mathbf{k}}\right| / b_{\mathbf{k}}$ WHERE $\mathbf{k} \in N^{r}$
}

\author{
GALEN R. SHORACK AND R. T. SMYTHE ${ }^{1}$
}

\begin{abstract}
Lemma 1 presents a powerful general inequality for $\max \left|S_{\mathbf{k}}\right| / b_{\mathbf{k}}$. This is applied in multidimensional time to sums of independent random variables and martingales to yield both old and new inequalities of the Doob, Hájek-Rényi, Skorokhod and Marcinkiewicz-Zygmund types.

A brief application is made to the partial sum process.
\end{abstract}

1. A general inequality. Let $N$ denote the positive integers; for $r \geq 1$ let $N^{r}$ denote the set of $r$-tuples $\mathbf{k}=\left(k_{1}, \ldots, k_{r}\right), k_{i} \in N$. Let $\left\{X_{\mathbf{k}}\right\}_{\mathbf{k} \in N^{r}}$ be random variables. Let $\left\{b_{\mathbf{k}}\right\}_{\mathbf{k} \in N^{r}}$ be a set of positive constants such that

$$
\Delta b_{\mathbf{k}} \geq 0 \text { for all } \mathbf{k} \geq \mathbf{1}
$$

here $\Delta b_{\mathbf{k}}$ is the usual $r$-dimensional differencing of $b_{\mathbf{k}}$ around the $2^{r}$ points of $N^{r}$ neighboring $\mathbf{k}$ which are $\leq \mathbf{k}$ in the usual coordinatewise ordering, and $\mathbf{1}$ $=(1, \ldots, 1)$. Define

$$
S_{\mathbf{n}}=\sum_{\mathbf{k} \leq \mathbf{n}} X_{\mathbf{k}}, \quad M_{\mathbf{n}}=\max _{\mathbf{k} \leq \mathbf{n}}\left|S_{\mathbf{k}}\right|, \quad Y_{\mathbf{k}}=X_{\mathbf{k}} / b_{\mathbf{k}}, \quad T_{\mathbf{n}}=\sum_{\mathbf{k} \leq \mathbf{n}} Y_{\mathbf{k}} .
$$

The following lemma is useful for proving inequalities for $\max \left|S_{\mathbf{k}}\right| / b_{\mathbf{k}}$.

LEMMA 1. If the positive $b_{\mathbf{k}}$ satisfy (1), then for arbitrary $X_{\mathbf{k}}$,

$$
\max _{\mathbf{k} \leq \mathbf{n}}\left|S_{\mathbf{k}}\right| / b_{\mathbf{k}} \leq 2^{r} \max _{\mathbf{k} \leq \mathbf{n}}\left|T_{\mathbf{k}}\right|
$$

If all $T_{\mathbf{k}}$ are $\geq 0$, we may replace $2^{r}$ by $2^{r-1}$.

Proof. Define $b_{\mathbf{k}}$ and $X_{\mathbf{k}}$ to be zero if some $k_{i}=0,1 \leq i \leq r$. Then (cf. [13]):

$$
S_{\mathbf{k}}=\sum_{\mathbf{j} \leq \mathbf{k}} b_{\mathbf{j}}\left(\Delta T_{\mathbf{j}}\right)=\sum_{\mathbf{j} \leq \mathbf{k}}\left(\Delta T_{\mathbf{j}}\right) \sum_{\mathbf{i} \leq \mathbf{j}}\left(\Delta b_{\mathbf{i}}\right)=\sum_{\mathbf{i} \leq \mathbf{k}} T_{\mathbf{i k}}\left(\Delta b_{\mathbf{i}}\right)
$$

where $T_{\mathbf{i k}}=\sum_{\mathbf{i} \leq \mathbf{j} \leq \mathbf{k}} Y_{\mathbf{j}}$. Note that each $T_{\mathbf{i k}}$ is formed by differencing $2^{r}$ sums of the type $T_{\mathbf{j}}$. Since $\sum_{\mathbf{i} \leq \mathbf{k}}\left(\Delta b_{\mathbf{i}} / b_{\mathbf{k}}\right)=1$ with each $\Delta b_{\mathbf{i}} \geq 0$ we thus have

$$
\begin{aligned}
\max _{\mathbf{k} \leq \mathbf{n}}\left|S_{\mathbf{k}}\right| / b_{\mathbf{k}} & \leq \max _{\mathbf{k} \leq \mathbf{n}} \sum_{\mathbf{i} \leq \mathbf{k}}\left(\Delta b_{\mathbf{i}}\right)\left|T_{\mathbf{i k}}\right| / b_{\mathbf{k}} \\
& \leq 1 \max _{\mathbf{k} \leq 1 \mathbf{n}} \max _{\mathbf{i} \leq \mathbf{k}}\left|T_{\mathbf{i k}}\right| \leq 2^{r} \max _{\mathbf{k} \leq \mathbf{n}}\left|T_{\mathbf{k}}\right|
\end{aligned}
$$

Received by the editors August 7, 1974 and, in revised form, November 11, 1974.

AMS (MOS) subject classifications (1970). Primary 60G45, 60G50; Secondary 60B10.

Key words and phrases. Hájek-Rényi and Skorokhod inequalities, multidimensional time, martingales, partial sum process.

${ }^{1}$ Research supported in part by the National Science Foundation under Grant GP-31361X and GP43328.

(C) American Mathematical Society 1976 
Clearly $2^{r-1}$ works if $T_{\mathbf{k}} \geq 0$ for all $\mathbf{k}$.

In the next section we apply Lemma 1 to deduce results concerning $\max \left|S_{\mathbf{k}}\right| / b_{\mathbf{k}}$ from both known and new inequalities for $M_{\mathbf{n}}$.

2. Martingale inequalities in $N^{r}$. Let $\left\{\mathscr{F}_{\mathbf{k}}\right\}_{\mathbf{k} \in N^{r}}$ be a collection of $\boldsymbol{\sigma}$-fields such that $\mathbf{k} \leq \mathbf{n}$ implies $\mathscr{F}_{\mathbf{k}} \subset \mathscr{F}_{\mathbf{n}}$ and let $Z_{\mathbf{k}}$ be $\mathscr{F}_{\mathbf{k}}$-integrable. We say that $\left(Z_{\mathbf{k}}\right.$, $\mathscr{F}_{\mathbf{k}}$ ) is a martingale* $\left(\mathrm{MG}^{*}\right)$ (resp., submartingale* $\left(\mathrm{SMG}^{*}\right)$ ) if

$$
E\left(Z_{\mathbf{k}} \mid \mathscr{F}_{\mathbf{i}}\right)=(\text { resp., } \geq) Z_{\mathbf{i} \wedge \mathbf{k}} \text { for all } \mathbf{i}, \mathbf{k} .
$$

When $r=1$ this is the usual definition of a MG or SMG, but for $r>1$ it is stronger. Let $S_{\mathbf{k}}$ be as in (2); note that

(i) if $\left(S_{\mathbf{k}}, \mathscr{F}_{\mathbf{k}}\right)$ is a $\mathrm{MG}^{*}$, so is $\left(T_{\mathbf{k}}, \mathscr{F}_{\mathbf{k}}\right)$;

(ii) if $\hat{\mathbf{k}}=\left(k_{1}, \ldots, k_{r-1}\right)$ and $L_{\hat{\mathbf{k}}} \equiv \max _{k_{r} \leq n_{r}}\left|S_{\left(\hat{\mathbf{k}}_{2} k_{r}\right)}\right|$, then $\left(L_{\hat{\mathbf{k}}}, \mathscr{F}_{\left(\hat{\mathbf{k}}, n_{r}\right)}\right)$ is an $r-1$ dimensional $\mathrm{SMG}^{*}$, provided that $\left(S_{\mathbf{k}}, \mathscr{F}_{\mathbf{k}}\right)$ is a $\mathrm{MG}^{*}$ or nonnegative $\mathrm{SMG}^{*}$.

To show (ii), note that $\left|S_{\mathbf{k}}\right|$ is a $\mathrm{SMG}^{*}$; and thus

$$
\begin{aligned}
E\left(L_{\hat{\mathbf{k}}} \mid \mathscr{F}_{\left(\hat{\mathbf{i}}, n_{r}\right)}\right) & \geq \max _{k_{r} \leq n_{r}} E\left(\left|S_{\left(\hat{\mathbf{k}}, k_{r}\right)}\right| \mid \mathscr{F}_{\left(\hat{\mathbf{i}}, n_{r}\right)}\right) \\
& \geq \max _{k_{r} \leq n_{r}}\left|S_{\left(\hat{\mathbf{k}} \wedge \hat{\mathbf{i}}, k_{r}\right)}\right|=L_{\hat{\mathbf{k}} \wedge \hat{\mathbf{i}}} .
\end{aligned}
$$

We assume henceforth that $\left(S_{\mathbf{k}}, \mathscr{F}_{\mathbf{k}}\right)$ is a $\mathrm{MG}^{*}$ or nonnegative $\mathrm{SMG}^{*}$. An inductive proof based on (ii) easily yields

$$
E\left\{M_{\mathbf{n}}^{p}\right\} \leq(p /(p-1))^{r p} E\left|S_{\mathbf{n}}\right|^{p}, \quad p>1, r \geq 1 .
$$

This is due to Doob (1953, p. 317) when $r=1$ and to Cairoli (1970) when $r>1$ (see Wichura (1969) in case of independence and $p=2$ ). Another inductive argument based on Doob's upcrossing inequality leads to the new inequality

$$
\begin{aligned}
P\left\{M_{\mathbf{n}} \geq 2^{r} \lambda\right\} & \leq \lambda^{-1} \int_{\lambda}^{\infty}(\log u)^{r-1} P\left\{\left|S_{\mathbf{n}}\right| \geq u\right\} d u \\
& \leq \lambda^{-1} \int_{\left\{\left|S_{\mathbf{n}}\right| \geq \lambda\right\}}\left|S_{\mathbf{n}}\right|\left(\log \left|S_{\mathbf{n}}\right|\right)^{r-1} d P, \quad r \geq 1, \lambda \geq 1 .
\end{aligned}
$$

is a Skorokhod-type improvement of another of Cairoli's inequalities [5, p. 3]; see $\$ 4$ for the proof of $(5)$.

In this paragraph suppose the $X_{\mathbf{k}}$ are independent with zero means. Additional inequalities are then possible. Wichura (1969) has proved the following Skorokhod-type inequality:

$$
\begin{array}{r}
P\left\{M_{\mathbf{n}} \geq 2^{r} \lambda\right\} \leq\left(1-\operatorname{Var}\left[S_{\mathbf{n}}\right] / \lambda^{2}\right)^{-r} P\left\{\left|S_{\mathbf{n}}\right| \geq \lambda\right\}, \\
r \geq 1, \lambda^{2} \geq \operatorname{Var}\left[S_{\mathbf{n}}\right] .
\end{array}
$$

A very interesting inequality involving only the first moment is

$$
P\left\{M_{\mathbf{n}} \geq \lambda\right\} \leq \lambda^{-1} E\left\{M_{\mathbf{n}}\right\} \leq \lambda^{-1}\left(2^{r+1}+1\right) E\left|S_{\mathbf{n}}\right|, \quad r \geq 1, \lambda>0 .
$$

For the case $r=1$, see Hunt (1966, p. 69); for $r=2$ the result has been 
established independently by Gabriel (1974). A proof of (7) is given in $\S 4$.

By applying Lemma 1 in conjunction with the above inequalities, some useful inequalities for $\max \left|S_{\mathbf{k}}\right| / b_{\mathbf{k}}$ can be derived. Applying Chebyshev's inequality along with Lemma 1 and (4) we get

$$
\begin{aligned}
P\left\{\max _{\mathbf{k} \leq \mathbf{n}}\left|S_{\mathbf{k}}\right| b_{\mathbf{k}}\right. & \geq \lambda\} \leq \lambda^{-p} 2^{r p} E\left(\max _{\mathbf{k} \leq \mathbf{n}}\left|T_{\mathbf{k}}\right|^{p}\right) \\
& \leq \lambda^{-p} 2^{r p}(p /(p-1))^{r p} E\left(\left|T_{\mathbf{n}}\right|^{p}\right), \quad r \geq 1, \lambda>0, p>1, \\
& =\lambda^{-2} 4^{2 r} \sum_{\mathbf{k} \leq \mathbf{n}} \operatorname{Var}\left[X_{\mathbf{k}}\right] b_{\mathbf{k}}^{2} \quad \text { when } p=2
\end{aligned}
$$

for any $\mathrm{MG}^{*}$ (cf. Smythe (1974)).

Now suppose in this paragraph that the $X_{\mathbf{k}}$ are independent with zero means. Application of Lemma 1 to (6) gives

$$
\begin{array}{r}
P\left\{\max _{\mathbf{k} \leq \mathbf{n}}\left|S_{\mathbf{k}}\right| / b_{\mathbf{k}} \geq 4^{r} \lambda\right\} \leq\left(1-\operatorname{Var}\left[T_{\mathbf{n}}\right] / \lambda^{2}\right)^{-r} P\left\{\left|T_{\mathbf{n}}\right| \geq \lambda\right\}, \\
r \geq 1, \lambda^{2} \geq \operatorname{Var}\left[T_{\mathbf{n}}\right] .
\end{array}
$$

This inequality seems to be new, even for $r=1$. Application of Lemma 1 to (7) gives

$$
\begin{aligned}
P\left\{\max _{\mathbf{k} \leq \mathbf{n}}\left|S_{\mathbf{k}}\right| b_{\mathbf{k}} \geq \lambda\right\} & \leq \lambda^{-1} 2^{r} E\left\{\max _{\mathbf{k} \leq \mathbf{n}}\left|T_{\mathbf{k}}\right|\right\} \\
& \leq \lambda^{-1} 2^{r}\left(2^{r+1}+1\right) E\left|T_{\mathbf{n}}\right|, \quad r \geq 1, \lambda>0 .
\end{aligned}
$$

Combining Lemma 1 with (8) and (10) we obtain

$$
\begin{aligned}
& E\left(\max _{\mathbf{k} \leq \mathbf{n}}\left|S_{\mathbf{k}}\right|^{p} / b_{\mathbf{k}}^{p}\right) \leq C_{p, r} E\left|T_{\mathbf{n}}\right|^{p} \leq 2 C_{p, r} \sum_{\mathbf{k} \leq \mathbf{n}} E\left|X_{\mathbf{k}}\right|^{p} b_{\mathbf{k}}^{p}, \\
& r \geq 1,1 \leq p \leq 2 ;
\end{aligned}
$$

where the second inequality for $1 \leq p \leq 2$ follows from von Bahr and Esseen (1965). The constant $C_{p, r}$ equals $2^{r}\left(2^{r+1}+1\right)$ for $p=1$ and equals $(2 p /(p-1))^{r p}$ for $1<p \leq 2$. Equation (11) generalizes some results for $r=$ 1 due to Bickel (1970) and Dunnage (1972).

In closing this section, we remark first that for $r=1$, the BirnbaumMarshall-Chow inequality [3], [6] can be strengthened along the lines of (5), and second that Lemma 1 can be applied to various continuous parameter processes by a passage to the limit.

3. Applications. We indicate here some applications of the results of $\$ 2$.

(A) Let $\mathbf{t}=\left(t_{1}, \ldots, t_{r}\right), I \equiv\{\mathbf{t}: 0 \leq \mathbf{t} \leq 1\}$. Define

$$
Z_{\mathbf{n}}(\mathbf{t})=\sum_{\mathbf{k} \leq \mathbf{n t}} X_{\mathbf{k}} /\left(\sigma^{2} n_{1} \cdots n_{r}\right)^{1 / 2}
$$

where the $X_{\mathbf{k}}$ are i.i.d. with mean 0 , variance $\sigma^{2}$, and $\mathbf{n t}=\left(n_{1} t_{1}, \ldots, n_{r} t_{r}\right)$. Let $q$ be a continuous function defined on $I$ such that

$$
\int_{0}^{1}[q(\mathbf{t})]^{-2} \prod_{1}^{r} d t_{i}<\infty \quad \text { and } \quad \Delta q \geq 0
$$


where $\Delta q$ denotes the obvious analog of (1). Let $\rho$ denote the supremum metric, and let

$$
\rho_{q}(f, g) \equiv \sup _{\mathbf{t} \in I}|f(\mathbf{t})-g(\mathbf{t})| / q(\mathbf{t}) .
$$

Let $D$ denote the set of all functions on $I$ which are "continuous from above with limits from below", as made precise in [2]. Let $Z$ denote the Wiener process in $r$-dimensional time (see [16], for example). Then

$$
Z_{\mathbf{n}} \Rightarrow Z \text { on }\left(D, \rho_{q}\right) \quad \text { as } \mathbf{n} \rightarrow \infty ;
$$

where " $\Rightarrow$ " means that $E\left[\psi\left(Z_{\mathbf{n}}\right)\right] \rightarrow E[\psi(Z)]$ as $\mathbf{n} \rightarrow \infty$ for all bounded functions $\psi$ which are $\rho_{q}$-continuous on $D$ and are measurable with respect to the $\sigma$-field generated by the finite-dimensional subsets of $D$. Our proof will clearly carry over to the non-i.i.d case considered in [2], the only problem being added notation.

Proof of (14). $Z_{\mathbf{n}} \Rightarrow Z$ on $(D, \rho)$ is known from [2]; and as in [12], we may suppose $\rho\left(Z_{\mathbf{n}}, Z\right) \stackrel{\mu}{\longrightarrow} 0$ by using different versions of the processes. For a real $\theta$ to be specified below, let $\boldsymbol{\theta}_{i}=(1,1, \ldots, 1, \theta, 1, \ldots, 1)$ for $1 \leq i \leq r$. Let $b_{\mathbf{k}}=q(\mathbf{k} / \mathbf{n})$, where $\mathbf{k} / \mathbf{n}=\left(k_{1} / n_{1}, \ldots, k_{r} / n_{r}\right)$ and $\mathbf{n}$ is fixed for the moment. Then for any $\delta>0$ we have from (8) that

$$
\begin{aligned}
P\left[\sup _{\mathbf{0} \leq \mathbf{t} \leq \boldsymbol{\theta}_{i}} \frac{\left|Z_{\mathbf{n}}(\mathbf{t})\right|}{q(\mathbf{t})} \geq \delta\right] & \leq P\left[\max _{\mathbf{k} \leq \mathbf{n} \boldsymbol{\theta}_{i}} \frac{\left|S_{\mathbf{k}}\right|}{b_{\mathbf{k}}} \geq \delta \sigma \sqrt{n_{1} \cdots n_{r}} / 2\right] \\
& \leq\left(\frac{4^{2 r+1}}{\delta^{2} n_{1} \cdots n_{r}}\right) \sum_{\mathbf{k} \leq \mathbf{n} \boldsymbol{\theta}_{i}} b_{\mathbf{k}}^{-2} \\
& \leq\left(\frac{4^{2 r+1}}{\delta^{2}}\right) \int_{0}^{\boldsymbol{\theta}_{i}}[q(\mathbf{t})]^{-2} \prod_{1}^{r} d t_{i} .
\end{aligned}
$$

From (13) we may choose $\theta$ to make the last term above $<\delta$. Using a limiting argument based on the continuity of the Brownian process, it is clear that the same inequality can be derived for $Z$. Since

$$
\rho_{q}\left(Z_{\mathbf{n}}, Z\right) \leq \sum_{i=1}^{r} \sup _{\mathbf{0} \leq \mathbf{t} \leq \boldsymbol{\theta}_{i}} \frac{\left|Z_{\mathbf{n}}(\mathbf{t})\right|}{q(\mathbf{t})}+\sum_{i=1}^{r} \sup _{\mathbf{0} \leq \mathbf{t} \leq \boldsymbol{\theta}_{i}} \frac{|Z(\mathbf{t})|}{q(\mathbf{t})}+\frac{\rho\left(Z_{\mathbf{n}}, Z\right)}{q(\boldsymbol{\theta} \mathbf{1})},
$$

we have

$$
P\left(\rho_{q}\left(Z_{\mathbf{n}}, Z\right) \geq(2 r+1) \delta\right) \leq 2 r \delta+P\left(\rho\left(Z_{\mathbf{n}}, Z\right) \geq \delta q(\theta \mathbf{1})\right) \leq(2 r+1) \delta
$$

for $\mathbf{n}$ exceeding some $\mathbf{n}_{\delta}$. Thus $\rho_{q}\left(Z_{\mathbf{n}}, Z\right) \stackrel{p}{\rightarrow} 0$; hence for the original processes, $Z_{\mathbf{n}} \Rightarrow Z$ on $\left(D, \rho_{q}\right)$. (Note the proof of Theorem 2.1 of [12].)

(B) One use of (6) is in establishing the tightness of the partial sum process, where one uses the asymptotic normality to suitably approximate $P\left(\left|S_{\mathbf{k}}\right| \geq \lambda\right)$. The same technique could be applied to (5) (cf. [4] for $r=1$ ); the integral of $\left|S_{\mathbf{k}}\right|\left(\log \left|S_{\mathbf{k}}\right|\right)^{r-1}$ over $[\lambda, \infty)$ may be approximated by its limiting normal value, because it is dominated by $S_{\mathbf{n}}^{2}$.

(C) Useful results for empirical processes based on Lemma 1 and (5) have 
been obtained in a Ph.D. thesis by Jon Wellner of the University of Washington.

(D) Inequality (8) has been used to establish strong laws of large numbers for i.i.d. random variables [13]; inequality (11) is used in [14] to establish a law of large numbers of the form $P\left\{\left|S_{\mathbf{k}}\right| / b_{\mathbf{k}}>\epsilon\right.$ i.o. $\}=0$ for nonidentically distributed $X_{\mathbf{k}}$.

\section{Proofs of (5) and (7).}

Proof of (5). Since $\left\{M_{n} \geq 2 \lambda\right\}$ implies, taking $S_{0} \equiv 0$, that either an upcrossing of $[\lambda, 2 \lambda]$ or a downcrossing of $[-\lambda,-2 \lambda]$ takes place, Doob's upcrossing inequality $[7$, p. 316$]$ yields (see also Brown (1971))

$$
\begin{aligned}
P\left\{M_{n} \geq 2 \lambda\right\} & \leq \lambda^{-1} \int_{\left\{\left|S_{n}\right| \geq \lambda\right\}}\left(\left|S_{n}\right|-\lambda\right) d P \\
& =\lambda^{-1} \int_{\lambda}^{\infty} P\left(\left|S_{n}\right| \geq t\right) d t, \quad r=1, \lambda>0 .
\end{aligned}
$$

Now note that (ii) of $\$ 2$ extends easily to show that $L_{k} \equiv \max _{\hat{\mathbf{i}} \leq \hat{\mathbf{k}} \leq \hat{\mathbf{n}}}\left|S_{\hat{\mathbf{k}}, k}\right|, 1$ $\leq k \leq n_{r}$, is a one-dimensional SMG with respect to the $\boldsymbol{\sigma}$-fields $\mathscr{F}_{\hat{\mathbf{n}}, k}$. Assuming the validity of (5) in dimension $r-1$,

$$
\begin{aligned}
P\left\{M_{\mathbf{n}} \geq 2^{r} \lambda\right\}= & P\left(\max _{1 \leq k \leq n_{r}} L_{k} \geq 2^{r} \lambda\right) \\
\leq & \left(2^{r-1} \lambda\right)^{-1} \int_{2^{r-1} \lambda}^{\infty} P\left(L_{n_{r}} \geq t\right) d t \quad(\text { by }(15)) \\
= & \lambda^{-1} \int_{\lambda}^{\infty} P\left(L_{n_{r}} \geq 2^{r-1} s\right) d s \\
\leq & \lambda^{-1} \int_{\lambda}^{\infty} s^{-1} \int_{s}^{\infty}\left(\log ^{r-2} u\right) P\left\{\left|S_{\mathbf{n}}\right| \geq u\right\} d u d s \\
= & \lambda^{-1} \int_{\lambda}^{\infty}\left(\log ^{r-1} u\right) P\left\{\left|S_{\mathbf{n}}\right| \geq u\right\} d u \\
& -\frac{\ln \lambda}{\lambda} \int_{\lambda}^{\infty}\left(\log ^{r-2} u\right) P\left\{\left|S_{\mathbf{n}}\right| \geq u\right\} d u \\
\leq & \lambda^{-1} \int_{\lambda}^{\infty}\left(\log ^{r-1} u\right) P\left\{\left|S_{\mathbf{n}}\right| \geq u\right\} d u \quad \text { for } \lambda \geq 1 .
\end{aligned}
$$

The second inequality of (5) is easily verified by induction, using the relation

$$
\begin{aligned}
\int_{\{Z \geq \lambda\}} Z(\log Z)^{r} d P= & \lambda(\log \lambda)^{r} P(Z \geq \lambda) \\
& +\int_{\lambda}^{\infty} P(Z \geq t) \frac{d}{d t}\left[t(\log t)^{r}\right] d t, \quad Z \geq 0, \lambda>0 .
\end{aligned}
$$

Proof of (7). The proof rests upon the following result (see, e.g., [11, Theorem 1]): If the $X_{\mathbf{k}}$ are independent and symmetric, then

$$
P\left\{\sup _{\mathbf{k} \leq \mathbf{n}}\left|S_{\mathbf{k}}\right| \geq \lambda\right\} \leq 2^{r} P\left\{\left|S_{\mathbf{n}}\right| \geq \lambda\right\}, \quad r \geq 1, \lambda>0 .
$$

One next observes that for symmetric $X_{\mathbf{k}}$, using (16), 


$$
E\left\{M_{\mathbf{n}}\right\}=\int_{0}^{\infty} P\left\{M_{\mathbf{n}} \geq \lambda\right\} d \lambda \leq 2^{r} \int_{0}^{\infty} P\left\{\left|S_{\mathbf{n}}\right| \geq \lambda\right\} d \lambda \leq 2^{r} E\left|S_{\mathbf{n}}\right| .
$$

For $X_{\mathbf{k}}$ not necessarily symmetric, one symmetrizes the $X_{\mathbf{k}}$, applies (16) and (17), and then "desymmetrizes", as in Hunt (1966), to give the result in (7).

\section{REFERENCES}

1. P. Bickel (1970), A Hájek-Rényi extension of Lévy's inequality and some applications, Acta Math. Acad. Sci. Hungar. 21, 199-206. MR 41 \#6266.

2. P. Bickel and M. Wichura (1971), Convergence criteria for multiparameter stochastic processes and some applications, Ann. Math. Statist. 42, 1656-1670.

3. Z. W. Birnbaum and A. W. Marshall (1961), Some multivariate Chebyshev inequalities with extensions to continuous parameter processes, Ann. Math. Statist. 32, 687-703. MR 26 \#615.

4. B. M. Brown (1971), Martingale central limit theorems, Ann. Math. Statist. 42, 59-66. MR 44 \#609.

5. R. Cairoli (1970), Une inégalité pour martingales à indices multiples et ses applications, Séminaire de Probabilités, IV (Univ. Strasbourg, 1968/69), Lecture Notes in Math., vol. 124, Springer-Verlag, Berlin, pp. 1-27. MR 42 \#5312.

6. Y. S. Chow (1960), A martingale inequality and the law of large numbers, Proc. Amer. Math. Soc. 11, $107-111$. MR 22 \#3045.

7. J. L. Doob (1953), Stochastic processes, Wiley, New York; Chapman \& Hall, London. MR $15,445$.

8. J. E. A. Dunnage (1972), Extensions of the Hájek-Rényi inequality to moments of higher order, Proc. Cambridge Philos. Soc. 72, 67-75. MR 48 \#108.

9. J.-P. Gabriel (1974), Lois des grands nombres, séries, et martingales à deux indices, C. R. Acad. Sci. Paris Sér. A-B 279, A169-A171.

10. G. A. Hunt (1966), Martingales et processus de Markov, Monographies de la Société Mathématique de France, no. 1, Dunod, Paris. MR 35 \#2333.

11. S. R. Paranjape and C. Park (1973), Laws of iterated logarithm of multiparameter Wiener processes, J. Multivariate Anal. 3, 132-136. MR 48 \#5194.

12. R. Pyke and G. R. Shorack (1968), Weak convergence of a two-sample empirical process and a new approach to Chernoff-Savage theorems, Ann. Math. Statist. 39, 755-771. MR 37 \#2357.

13. R. T. Smythe (1974), Sums of independent random variables on partially ordered sets, Ann. Probability 2, 906-917.

14. (1975), Ergodic properties of marked point processes in $R^{r}$, Ann. Inst. H. Poincaré 11 , $109-125$.

15. B. von Bahr and C. G. Esseen (1965), Inequalities for the rth absolute moment of a sum of random variables, $1 \leq r \leq 2$, Ann. Math. Statist. 36, 299-303. MR 30 \#645.

16. M. J. Wichura (1969), Inequalities with applications to the weak convergence of random processes with multi-dimensional time parameters, Ann. Math. Statist. 40, 681-687. MR 39 \# 7663.

Department of Mathematics, University of Washington, Seattle, Washington 98195 